\title{
Functional Monoplegia in the context of extensive deconditioning and spinal stenosis
}

Marie Halfman ${ }^{1 *}$ and Camille Halfman ${ }^{2}$

*Correspondence: mlhalfman@gmail.com

CrossMark

$\leftarrow$ Click for updates

'School of Health and Human Services, Nazareth College of Rochester, Rochester, NY, USA.

${ }^{2}$ Department of Emergency Medicine, SUNY Upstate Medical University, Syracuse, NY, USA.

\begin{abstract}
Case study of a patient (pt) presenting with positional loss of muscle tone and deep tendon reflex (DTR) of the left lower extremity (LLE). MRI identified lumbar spinal stenosis but failed to identify other disease processes accounting for the motor deficit. We hypothesized that an increase in sedentary lifestyle related to the stay at home order for the 2020 COVID-19 pandemic, superimposed on generalized deconditioning resulting in postural disturbance, led to anatomic dysfunction sufficient to block motor signals to the LLE. Conservative management including positioning, stretching, and manual therapy allowed the pt to demonstrate a return of active movement of the LLE against gravity in all major joints. This treatment protocol is presented as a novel, conservative approach to restoring functionality in a pt with a rare symptom of spinal stenosis: Functional monoplegia of a lower extremity. This case report additionally discusses the suspected functional impact of the COVID-19 stay at home order on the geriatric population.
\end{abstract}

Keywords: Geriatric, Spinal Stenosis, Monoplegia, COVID-19, Physical Therapy, Manual Therapy

\section{Introduction}

Lumbar spinal stenosis is the most significant cause of spinal surgery in adults over 65 , with 5.9 in 100 pts proceeding from first diagnosis to spinal fusion within one year of diagnosis. $19 \%$ of pts who undergo spinal surgery due to spinal stenosis require repeat surgery. There is no evidence to suggest that surgical management is superior to conservative management when comparing long term outcomes [1,2]. Spinal stenosis can refer to multiple anatomical disorders which may cause narrowing of the foreman including but not limited to degenerative spondylosis, fracture of the pars interarticularis, space-occupying lesions, post-surgical fibrosis, ankylosing spondylitis, other rheumatological or skeletal diseases. Lumbar radiculopathy is widely considered to be caused by stenosis of the lateral recess and neural foramen. Spinal stenosis uncommonly impacts motor control for the lower extremity, with leg weakness reported in just $33 \%-51 \%$ of cases $[3,4]$. Due to the lack of evidence to support surgical intervention, protocols for conservate management should be investigated as the first choice in treatment to prevent unwanted side effects of surgical intervention.

\section{Case Presentation}

An 86 year old female with past medical history of hypertension, lymphedema of the left upper extremity, a sequela of prior mastectomy for cancerous tissue removal, presented to a skilled nursing facility (SNF) after an unwitnessed ground level mechanical fall in the home. The immediate post fall assessment did not identify major injury and she was able to ambulate with assistance after being assisted to a standing position. The next morning, the pt was unable to safely transition out of bed due to weakness and lower extremity instability. She was subsequently diagnosed with the following during her acute stay: Orthostatic hypotension, spinal stenosis of the lumbar spine, generalized weakness.

Upon initial assessment the patient was assessed to be a frail elderly female with significant anxiety and fear of recurrent falls, requiring at least $75 \%$ physical assistance for functional mobility including bed mobility (rolling side to side, supine <> sit and scooting), sit to stand and stand pivot transfers, unable to ambulate at the time of the initial assessment. Manual Muscle Testing (MMT) was grossly 2+/5 for hip flexion, hip extension, 
knee extension, knee flexion, ankle plantar flexion and dorsiflexion of bilateral lower extremities (BLE). Sensation testing was diminished but otherwise unremarkable. Of note, while this pt did demonstrate a drop in blood pressure consistent with a diagnosis of orthostatic hypotension during her hospitalization, she did not demonstrate recurrent events during assessments in the SNF setting. Despite demonstration of $2+/ 5$ MMT on initial assessment, pt would inconsistently present with MMT of $0 / 5$ for LLE. A pattern was observed where this resident would be able to demonstrate muscle contraction immediately upon rising from a supine position, but be unable to produce significant contraction if she had been sitting in a wheelchair or recliner prior to assessment. The pt's presentation was isolated as a functional monoplegia for the LLE most significantly in the $L 3$ and caudal myotomes.

\section{Assessment}

MRI for lumbar spine (+) for spinal stenosis (+) thomas test indicative of iliopsoas and rectus femori (RF) shortening. Pain with palpation of the sacroiliac crests.

\section{Intervention}

The pt was placed in a position of posterior pelvic tilt via maximally tolerated flexion of the right lower extremity (RLE) in the supine position. While she was cued to perform abdominal bracing to reverse the normal lumbar lordosis, she was unable to sustain a contraction sufficient to participate in pelvic stabilization and manual stabilization was provided. LLE was slowly lowered into a position for iliopsoas and RF stretch with continued manual stabilization of the pelvis. Manual stabilization of the pelvis was deemed critical at this juncture as the pt was unable to demonstrate sufficient muscle contraction for internal stabilization. After sustaining the stretch for at least 5 minutes, the pt was provided with tapping technique to promote neuromuscular recruitment of the knee extension and plantar flexion musculature, while sustaining the aforementioned stretch. Within 7 minutes of sustained stretch, the pt would progress from demonstrating no palpable muscle contraction to demonstrating up to $50 \%$ available ROM against gravity for all major muscle groups in all major planes. She would maintain this movement for up to ten minutes after stretch cessation. Her plan of care was shifted to promote strengthening of the abdominal and gluteal muscles, in addition to strengthening the spinal intrinsics to maximize postural stability, with positioning to allow for passive stretch of LLE hip flexors to pt tolerance. Pt's ability to walk after sitting in a recliner gradually improved, ultimately she was withdrawn from the SNF and lost to follow up.

\section{Discussion}

This pt presented with a complete loss of muscle tone in a single limb dependent on spinal positioning. Per her report, she had given up usual daily activities of walking around the block, shopping, and working in her yard due to the stay at home order of the 2020 COVID-19 pandemic. She reported a significant increase in time spent in sitting and a lack of weight bearing exercise in the home to compensate for the sudden increase in sedentary lifestyle. A flexion contracture of the hip flexors, primarily iliopsoas, may result in compensatory hyperlordosis of the lumbar spine, which causes foraminal stenosis.' Multi-level weakness of thespinal intrinsics, abdominal and gluteal muscle weakness were thought to also contribute to the functional hyperflexion of the lumbar spine and resulting functional monoplegia.

\section{Conclusion}

Lumbar spinal stenosis is a common diagnosis impacting the geriatric population. Due to the COVID-19 stay at home order and sequelae including sharp expansion of sedentary lifestyle there is anticipated to be an increased incidence. Because of the lack of evidence to support a superior intervention management of lumbar spinal stenosis requires an interdisciplinary approach. This case study outlines a novel, conservative treatment to address arare symptom of spinal stenosis: Functional monoplegia of a lower extremity.

\section{Competing interests}

The authors declare that they have no competing interests.

Authors' contributions

\begin{tabular}{|l|c|c|}
\hline Authors' contributions & MH & CH \\
\hline Research concept and design & $\sqrt{ }$ & -- \\
\hline Collection and/or assembly of data & $\sqrt{ }$ & -- \\
\hline Data analysis and interpretation & $\sqrt{ }$ & -- \\
\hline Writing the article & $\sqrt{ }$ & $\sqrt{ }$ \\
\hline Critical revision of the article & $\sqrt{ }$ & $\sqrt{ }$ \\
\hline Final approval of article & $\sqrt{ }$ & $\sqrt{ }$ \\
\hline Statistical analysis & -- & -- \\
\hline
\end{tabular}

\section{Publication history}

Editor: Catherine Ortega, University of Texas Health Science Center, USA. Received: 30-May-2021 Final Revised: 15-July-2021

Accepted: 18-July-2021 Published: 01-Aug-2021

\section{References}

1. Wu L, Cruz R. Lumbar Spinal Stenosis. [Updated 2020 Dec 20]. In: StatPearls [Internet]. Treasure Island (FL): StatPearls Publishing 2021 Jan-. Available from $\cdot$ https://www.ncbi n m nih gov/books/NBK531493/

2. Zaina F, Tomkins-Lane C, Carragee E, Negrini S. Surgical Versus Nonsurgical Treatment for Lumbar Spinal Stenosis. Cochrane Database of Systemic Reviews2016 1:Art. No.: CD010264. https://www.cochrane ibrary.com/cdsr/doi/10 1002/14651858 CD010264 pub2/informatio n

3. Hilibrand A, Rand N. Degenerative Lumbar Stenosis: Diagnosis and Management. Journal of the American Academy of Orthopaedic Surgeons 1999 7(4):239-249. https://journals.Iww.com/jaaos/Fulltext/1999/07000/ Degenerative Lumbar Stenosis Diagnosis and.4.aspx

4. Iversen M, Katz J. Examination Findings and Self-Reported Walking Capacity inPatients With Lumbar Spinal Stenosis. Physical Therapy, 2001(81 :7):Pages 1296-1306, https://doi.org/10.1093/ptj/81.7.1296

5. Buckland A, Miyamoto R, Patel R, Slover J, Raiz A. Differentiating Hip Pathology From Lumbar Spine Pathology: Key Points of Evaluation and 
Halfman et al., Physical Therapy and Rehabilitation 2021,

http://www.hoajonline.com/journals/pdf/2055-2386-8-5.pdf

Management. J AmAcad Orthop Surg: 2017(25:2):e23-e34 https:// pubmed $\mathrm{ncbi} n \mathrm{~m}$ nih gov/28045713/

\section{Citation:}

Halfman $M$ and Halfman C. Functional Monoplegia in the context of extensive deconditioning and spinal stenosis.

Phys Ther Rehabil. 2021; 8:5.

http://dx.doi.org/10.7243/2055-2386-8-5 